\title{
Distribution of Alarin Immunoreactivity in the Mouse Brain
}

\author{
Nicole Eberhard - Christian Mayer • Radmila Santic • Ruben Peco Navio • \\ Andrea Wagner • Hans Christian Bauer • Guenther Sperk • Ulrich Boehm • \\ Barbara Kofler
}

Received: 24 March 2011 / Accepted: 5 May 2011 /Published online: 7 June 2011

(C) The Author(s) 2011. This article is published with open access at Springerlink.com

\begin{abstract}
Alarin is a 25 amino acid peptide that belongs to the galanin peptide family. It is derived from the galaninlike peptide gene by a splice variant, which excludes exon 3. Alarin was first identified in gangliocytes of neuroblastic tumors and later shown to have a vasoactive function in the skin. Recently, alarin was demonstrated to stimulate food intake as well as the hypothalamic-pituitary-gonadal axis in rodents, suggesting that it might be a neuromodulatory peptide in the brain. However, the individual neurons in the central nervous system that express alarin have not been identified. Here, we determined the distribution of alarinlike immunoreactivity (alarin-LI) in the adult murine brain.
\end{abstract}

N. Eberhard and C. Mayer contributed equally to this work.

N. Eberhard $\cdot$ R. Santic $\cdot$ B. Kofler $(\bowtie)$

Department of Pediatrics, Laura Bassi Centre of Expertise

THERAPEP, Research Program for Receptor Biochemistry and Tumormetabolism, Paracelsus Medical University,

Salzburg 5020, Austria

e-mail: b.kofler@salk.at

C. Mayer $\cdot$ R. P. Navio $\cdot$ U. Boehm $(\bowtie)$

Institute for Neural Signal Transduction,

Center for Molecular Neurobiology,

Hamburg 20253 Hamburg, Germany

e-mail: ulrich.boehm@zmnh.uni-hamburg.de

A. Wagner $\cdot$ H. C. Bauer

Department of Organismic Biology, University of Salzburg, Salzburg 5020, Austria

\footnotetext{
A. Wagner $\cdot$ H. C. Bauer

Department of Applied Cell Biology, Paracelsus Medical

University,

Salzburg 5020, Austria

G. Sperk

Department of Pharmacology, Medical University Innsbruck, Innsbruck 6020, Austria
}

The specificity of the antibody against alarin was demonstrated by the absence of labeling after pre-absorption of the antiserum with synthetic alarin peptide and in transgenic mouse brains lacking neurons expressing the GALP gene. Alarin-LI was observed in different areas of the murine brain. A high intensity of alarin-LI was detected in the accessory olfactory bulb, the medial preoptic area, the amygdala, different nuclei of the hypothalamus such as the arcuate nucleus and the ventromedial hypothalamic nucleus, the trigeminal complex, the locus coeruleus, the ventral chochlear nucleus, the facial nucleus, and the epithelial layer of the plexus choroideus. The distinct expression pattern of alarin in the adult mouse brain suggests potential functions in reproduction and metabolism.

Keywords Alarin · Galanin-like peptide · Neuropeptide · Central nervous system Cre recombinase .

Recombineering $\cdot$ Diphtheria toxin

\section{Introduction}

Alternative posttranscriptional splicing plays an important role in increasing the potential number of gene products, resulting in proteomic diversity especially in the central nervous system (Grabowski and Black 2001). The splice variants of one gene are often differentially regulated in a tissue- or developmental stage-specific manner (Enigk and Maimone 1999). Therefore, detailed information about the expression patterns of individual splice variants is needed to investigate their putative functions.

Galanin-like peptide (GALP) is an endogenous ligand of the galanin receptors, and its amino acid residues 9-21 show complete sequence homology with galanin (Ohtaki et 
al. 1999). GALP is characterized by differential splicing. One particular splice variant excludes exon 3 and results in a precursor protein that still harbors the signal sequence of prepro-GALP and the first five amino acids of the mature GALP peptide. Exclusion of exon 3 causes a frame shift and leads to a 20 amino acid sequence that does not show any homology to other proteins. Proteolytic processing of this splice variant results in a novel 25 amino acid neuropeptide, which was termed alarin because of its $\mathrm{N}$-terminal alanine and $\mathrm{C}$-terminal serine residues (Santic et al. 2006; Lang et al. 2007; Santic et al. 2007).

Alarin messenger RNA (mRNA) and peptide were originally detected in human neuroblastic tumors (Santic et al. 2006). Immunohistochemical analysis of tumor samples showed that alarin-like immunoreactivity (alarinLI) is specifically found in gangliocytes of differentiated neuroblastomas but not in undifferentiated tumor samples (Santic et al. 2006). Alarin-LI was also detected in human and murine skin (Santic et al. 2007). In the dermal vascular system, alarin peptide is present in pericytes of microvascular arterioles and venules and in layers of smooth muscle cells in larger vessels, but not in endothelial cells. In vivo experiments analyzing inflammatory edema formation in mice revealed a dose-dependent inhibitory effect of alarin on edema formation. This anti-edema effect is due to alarin's vasoconstrictor function (Santic et al. 2007).

Expression analysis revealed that alarin mRNA is also present in the central nervous system (Santic et al. 2007). Because GALP alters aspects of metabolism and reproduction, it is likely that alarin exerts similar effects via the brain. Recently, it was shown that alarin stimulates feeding behavior, increases body weight, and influences luteinizing hormone (LH) secretion in rats. Intercerebroventricular (i.c.v.) injection of alarin dose-dependently increased acute food intake (Van Der Kolk et al. 2010; Boughton et al. 2010). Furthermore, treatment with $1 \mathrm{nmol}$ alarin i.c. v. resulted in a significant increase in body weight after $24 \mathrm{~h}$, an effect that was not seen at doses over $5 \mathrm{nmol}$ (Van Der Kolk et al. 2010; Boughton et al. 2010). Male sexual behavior was not altered by alarin treatment in intact or castrated rats, but luteinizing hormone secretion was significantly increased in male rats (Van Der Kolk et al. 2010; Boughton et al. 2010). Furthermore, alarin is able to stimulate the release of gonadotropin-releasing hormone $(\mathrm{GnRH})$ in both hypothalamic explants and a hypothalamic cell line (Boughton et al. 2010). The effects of alarin seem to be mediated by specific alarin receptors because alarin lacks homology to galanin and is not able to compete with galanin for galanin receptors (Santic et al. 2007; Boughton et al. 2010).

Finally, i.c.v. alarin injection increased fos expression in the diencephalon and rhombencephalon of male rats. Fos-immunoreactivity was detected in hypothalamic nuclei of the paraventricular nucleus, the dorsomedial nucleus, and the arcuate nucleus, but the greatest induction of fos expression was observed in the solitary tract, the nucleus prepositus, the dorsal vestibular nucleus, and the olive nuclei (Van Der Kolk et al. 2010), indicating that receptors recognizing alarin are present in these brain areas.

To understand the neural circuitry underlying these effects, one first needs to identify the alarin-expressing neurons in the central nervous system. Here, we present the distribution of alarin-LI in the adult mouse brain.

\section{Materials and Methods}

\section{Alarin and GALP RT- and q-PCR}

To extract RNA from different brain areas, mice were euthanized, perfused with ice-cold ACSF $(125 \mathrm{mM} \mathrm{NaCl}$, $25 \mathrm{mM} \mathrm{NaHCO}, 1.25 \mathrm{mM} \mathrm{NaH} \mathrm{PO}_{4}, 2.5 \mathrm{mM} \mathrm{KCl}$, $0.05 \mathrm{mM} \mathrm{CaCl}_{2}, 6 \mathrm{mM} \mathrm{MgCl}_{2}, 2.5 \mathrm{mM}$ glucose, and $50 \mathrm{mM}$ sucrose) and decapitated. The brain was quickly removed, and coronal slices were cut on a Leica VT $1200 \mathrm{~S}$ vibratom.

For RT-PCR, different brain areas were punched out, and RNA was extracted using Trizol (Invitrogen, Darmstadt, Germany). For complementary DNA (cDNA) synthesis, a Transcriptor High Fidelity cDNA Synthesis Kit (Roche, Freiburg, Germany) was used following the manufacturer's instructions.

Because of low alarin/GALP transcript levels, a nested PCR was performed to analyze alarin/GALP expression in the different brain areas. The first PCR amplification comprised five cycles using TAQ DNA polymerase (Invitrogen, Darmstadt Germany) and primers 5'-cccaccettgtctctctccctaacctc-3' (exon 1) and 5'-gtggettcttccttgggaacattcttgt-3' (exon 6) for alarin/GALP and primers $5^{\prime}$-gaggagcaatgatcttgatcttcatggtg-3' (exon $2 / 3$ ) and $5^{\prime}$-ctaggcaccagggtgtgatggtgg-3' (exon 5/6) for $\alpha$-actin (control). The second amplification comprised 40 cycles using nested primers $5^{\prime}$-cctcattctcctcagaagctcacaaacc- $3^{\prime}$ (exon 1) and 5'-ccttgggaacattcttgtccagtatgtgc-3' (exon 6) for alarin/GALP and primers $5^{\prime}$-gacccagatcatgtttgagaccttcaac- $3^{\prime}$ (exon 3/4) and 5'-ccacaggattccatacccaagaaggaag-3' (exon 4/5) for $\alpha$-actin. PCR products were separated by gel electrophoresis and visualized with ethidium bromide. Successful amplification yielded a 354-bp alarin, a 413-bp GALP, and a 462-bp $\alpha$-actin amplicon (Fig. 1).

For quantitative RT-PCR (qPCR), the hypothalamic area between bregma 0.8 and -2.0 was punched out, and RNA was extracted using the RNeasy mini kit (Qiagen, Hilden, Germany). For cDNA synthesis, superscript II (Invitrogen) was used following the manufacturer's instructions. mRNA expression was analyzed using 
Fig. 1 Targeted ablation of alarin and GALP neurons. a Genetic strategy to express Cre recombinase in alarin and GALP neurons. b Transgenic mice harboring the AGALP-Cre BAC transgene were bred to the ROSA26-DTA strain

(Brockschnieder et al. 2006). In AGALP-Cre/R26-DTA mice, DTA is expressed after Cremediated excision of transcriptional "STOP" signals flanked by loxP sites, leading to death of the Cre-expressing cell. c Alarin/GALP mRNA levels were reduced by $\sim 91 \%$ in adult AGALP-Cre/R26-DTA animals $(n=2)$ compared to controls $(n=5)$. d Expression of GALP and alarin mRNA in different brain regions. RT-PCR analysis on total RNA extracted from different brain areas of wild-type mice. Both GALP (405 bp) and alarin (346 bp) mRNA are detected in the olfactory bulb, hypothalamus and brainstem, but not in the cortex $(O B$ olfactory bulb, MPA medial preoptic area, $A R C$ arcuate nucleus, $B S$ brainstem, $C x$ cortex). Squares in Fig. 4 indicate the regions punched out for RNA isolation

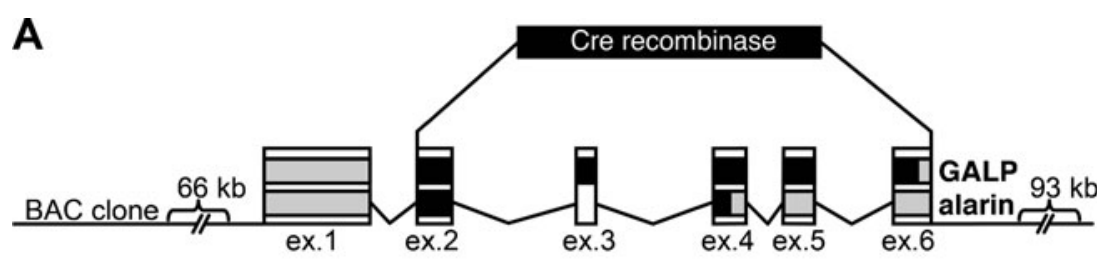

B

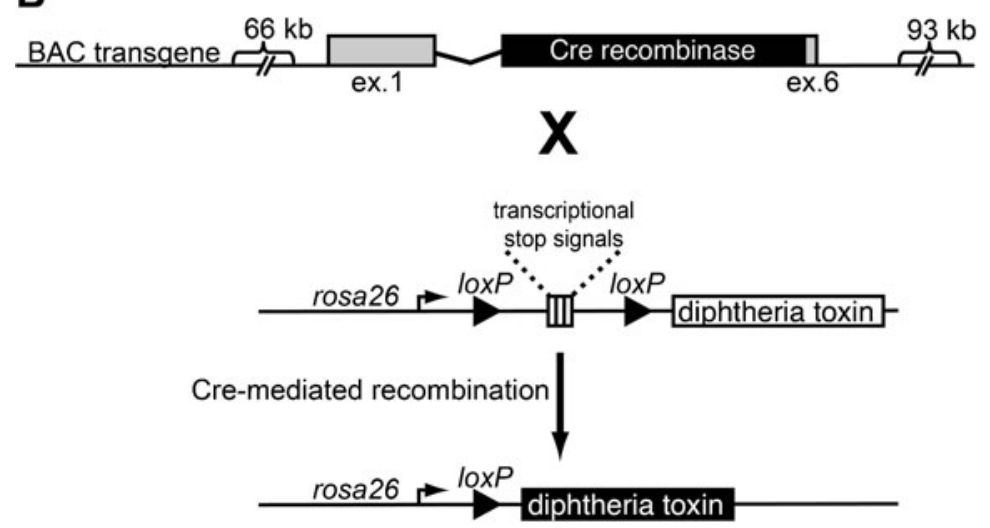

C
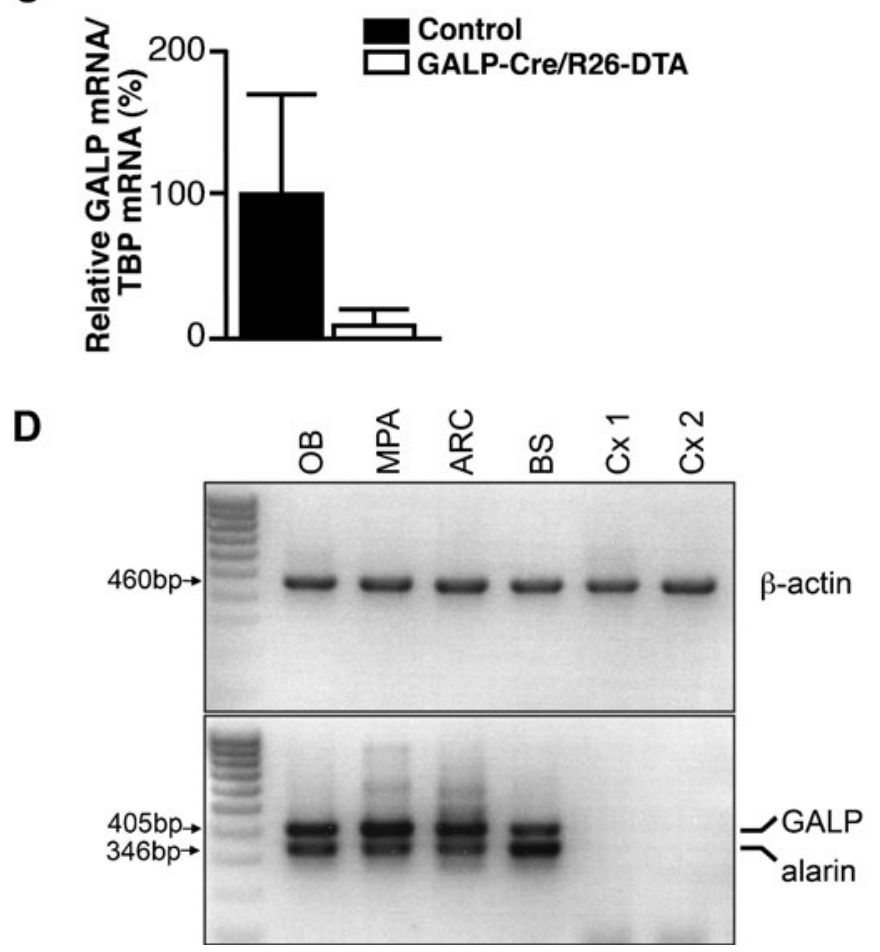

qPCR. cDNA was amplified using TaqMan Universal PCR Master Mix with TaqMan Assay-on-Demand kits for GALP (GALP, Mm00626135 m1) and TATA-box binding protein (TBP) to measure reference gene levels (TBP, Mm00446971_m1) (all from Applied Biosystems). Each reaction was run in triplicate. qPCR was performed on an ABI Prism 7700 sequence detector (Applied Biosystems). mRNA levels were calculated by a comparative method $\Delta(\Delta \mathrm{Ct}$ ), where $\Delta \mathrm{Ct}=\mathrm{Ct}$ (gene of interest $)-\mathrm{Ct}(\mathrm{TBP})$ and $\Delta(\Delta \mathrm{Ct})=\Delta \mathrm{Ct}$ (mutant) - mean $\Delta \mathrm{Ct}$ (control). Results are expressed as PCR-cycle differences in relative gene expression with respect to control.

Recombineering and Generation of AGALP-Cre Transgenic Mice

Bacterial artificial chromosome (BAC) RP23-404B17 (BACPAC Resources Center), comprising $\sim 66 \mathrm{~kb}$ of $5^{\prime}$ 
and $\sim 93 \mathrm{~kb}$ of $3^{\prime}$ flanking sequence of the GALP locus, was modified by homologous recombination in bacteria (recombineering) (Warming et al. 2005) (Fig. 1). A cDNA encoding Cre recombinase was PCR-amplified from plasmid pKO-IRES-CRE (Wen et al. 2008) using primers 5'-ccccaggectatggecacaaccatgtcc-3' and 5'ccccegccggetaatcgecatcttccagca-3' and placed upstream of an FRT-flanked neomycin resistance cassette in pBluescript (Stratagene, Amsterdam, The Netherlands). We then generated two 200-bp GALP-homology arms using primers GALP_start_f, 5'-ccgactgctggtccatagtgatcc-3'; GALP_start_r, 5'-gggggtcgaccetggagcggaaagagcacagcacacagc- $3^{\prime}$; GALP_stop_f, 5'-ggggactagtccatacctcactcctccttcgtt-3'; GALP_stop_r, 5'-cactgggtcatctctggggtccc-3', and placed them at both ends of this cassette. The homology arms were chosen such that the Cre cDNA would exactly replace the alarin/ GALP open reading frame from the ATG start to the stop codon (Fig. 1a). The final recombineering vector was linearized and electroporated into SW105 bacteria carrying BAC clone RP23-404B17 (Warming et al. 2005). Neomycin-resistant clones were screened by PCR and restriction digest analysis for correct targeting. Finally, we removed the neomycin cassette from recombinant BACs by arabinose-induced expression of FLP/FRT recombinase (Warming et al. 2005). The recombinant BAC was linearized using a loxP site in the vector backbone, purified and microinjected into pronuclei using standard methods.

Founder animals were backcrossed to $\mathrm{C} 57 \mathrm{BL} / 6 \mathrm{~J}$ animals to give alarin/GALP (AGALP)-Cre mice. For genotyping, genomic DNA from tail biopsies was prepared by proteinase K (Roche, Basel, Switzerland) digestion and subsequent purification with DNA Isolation Reagent for genomic DNA (AppliChem, Darmstadt, Germany). Mice were genotyped by PCR using internal Cre primers (5'-ggcgcggcaacaccattttt-3' and 5'ccgggetgecacgaccaa- $\left.3^{\prime}\right)$.

\section{Mice}

Animal care and experimental procedures were performed in accordance with the guidelines established by the animal welfare committee of the University of Hamburg. Mice were kept under a standard light/dark cycle with food and water ad libitum. AGALP-CRE mice were kept in a mixed $(129 / \mathrm{SvJ}$ and $\mathrm{C} 57 \mathrm{BL} / 6 \mathrm{~J})$ background.

To systemically ablate alarin and GALP neurons, we bred AGALP-Cre mice to the ROSA26-DTA (R26-DTA) strain, which carries a targeted insertion of the diphtheria toxin fragment $A$ (DTA) gene in the ROSA26 locus (Brockschnieder et al. 2006) (Fig. 1b). AGALP-Cre/R26DTA mice were born with expected Mendelian frequencies and were viable.
Preparation of Brain Samples

Four adult C57BL/6J and four AGALP-Cre/R26-DTA mice (3-4 months old) were anesthetized with ketamin/xylazine (Bayer, Leverkusen, Germany) and perfused transcardially with phosphate-buffered saline (PBS; $150 \mathrm{mM} \mathrm{NaCl}, 8 \mathrm{mM}$ $\left.\mathrm{Na}_{2} \mathrm{HPO}_{4}, 2 \mathrm{mM} \mathrm{KH} \mathrm{PO}_{4}, \mathrm{pH} 7.4\right)$ at $37^{\circ} \mathrm{C}$, followed by ice-cold $4 \%$ paraformaldehyde (Roth, Karlsruhe, Germany). After $2 \mathrm{~h}$ at $4{ }^{\circ} \mathrm{C}$, brains were removed from the skull and postfixed for $2 \mathrm{~h}$ at $4{ }^{\circ} \mathrm{C}$ in $4 \%$ paraformaldehyde. Brains were rinsed in PBS, transferred to $30 \%$ sucrose for $48 \mathrm{~h}$, embedded in tissue freezing medium OCT (Leica, Wetzlar, Germany) and frozen in isopentane.

\section{Generation of Anti-Alarin Antibody}

Rabbit polyclonal antisera were custom-made (Neo MPS, Strasbourg, France) using a synthetic murine alarin peptide 6-24-Cys (SSPFPPRPTRAGRETQLLRC) (Santic et al. 2007). Alarin 6-24 was coupled to the carrier protein keyhole limpet hemocyanin via a $\mathrm{C}$-terminal cysteine residue. Immunization was carried out on days 0 , 14,28 , and 56 . Affinity purification of the antiserum was carried out as described previously (Santic et al. 2006). Briefly, immunogenic murine alarin 6-24 was coupled to a HiTrap N-hydroxy-succinimide (NHS)-activated column according to the manufacturer's instructions (Amersham Biosciences, Buckinghamshire, UK). The polyclonal antialarin antiserum was loaded onto the column, and after several washing steps, the purified antibody was eluted and immediately neutralized using $20 \mathrm{mM}$ Tris- $\mathrm{HCl}$ (pH 9). Finally, the affinity-purified antibody was aliquoted and stored at $-80^{\circ} \mathrm{C}$ until use. To exclude crossreactivity of the antibody to galanin or GALP, dot blot analysis was performed. The affinity-purified antibody was only able to detect synthetic murine alarin peptide (NeoMPS, Strasbourg, France) but not galanin (Bachem, Bubendorf, Switzerland) and GALP (Bachem, Bubendorf, Switzerland).

\section{Immunohistochemistry}

For immunohistochemistry, $14-\mu \mathrm{m}$ coronal brain sections were cut on a cryostat, thaw-mounted on Superfrost ${ }^{\mathrm{TM}}$ slides (Menzel, Braunschweig, Germany), and stored at $-80^{\circ} \mathrm{C}$ until use. Sections were washed with PBS $(58 \mathrm{mM}$ $\left.\mathrm{Na}_{2} \mathrm{HPO}_{4}, 17 \mathrm{mM} \mathrm{NaH} \mathrm{PO}_{4}, 68 \mathrm{mM} \mathrm{NaCl}, \mathrm{pH} 7.4\right)$ and incubated in $0.5 \%$ Triton $\mathrm{X}-100$ in PBS for $10 \mathrm{~min}$ for antigen retrieval. Immunostaining was performed according to the protocol supplied with the DAKO ENVision+HRP Detection System (DAKO, Glostrup, Denmark), with modifications. Endogenous peroxidases were quenched using a peroxidase-blocking reagent for $5 \mathrm{~min}$. After washing in 

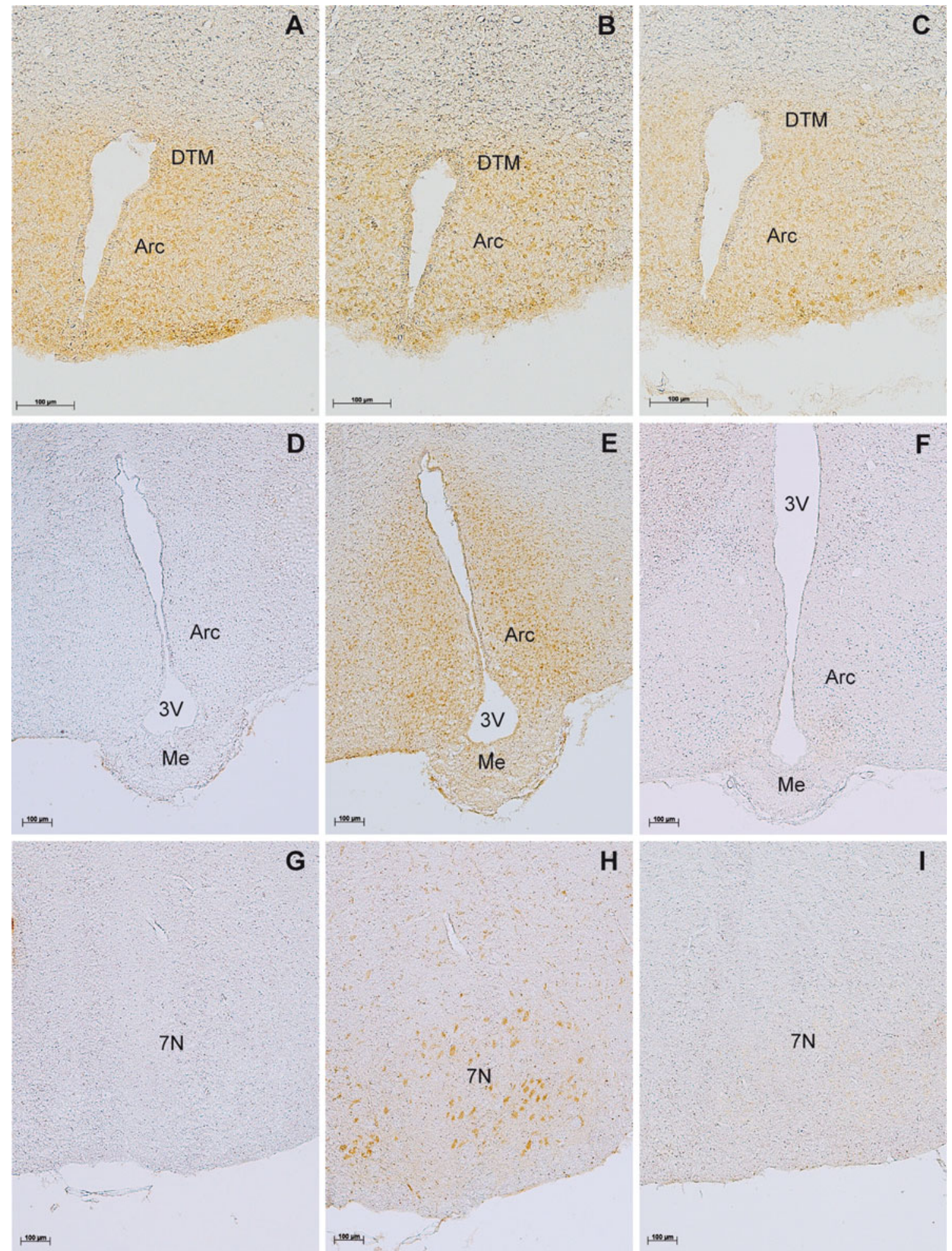

Fig. 2 Evaluation of the specificity of the rabbit-anti-alarin antibody. Alarin-LI in the arcuate nucleus $(\mathbf{a}-\mathbf{c}, \mathbf{e})$, the dorsal tuberomammilary nucleus (a-c), and the facial nucleus (h). Preabsoption with $10 \mu \mathrm{M}$ GALP peptide (a) or galanin (c) does not abolish staining, whereas

PBS-T ( $58 \mathrm{mM} \mathrm{Na}_{2} \mathrm{HPO}_{4}, 17 \mathrm{mM} \mathrm{NaH}_{2} \mathrm{PO}_{4}, 68 \mathrm{mM} \mathrm{NaCl}$, $0.5 \%$ Tween 20, $\mathrm{pH} 7.4$ ), nonspecific binding sites were preabsorption of the antibody with $10 \mu \mathrm{M}$ alarin completely abolishes alarin-LI (d, g). Alarin-LI is lacking in the arcuate (f) and the facial nucleus (i) of transgenic AGALP-Cre/R26-DTA mice. Abbreviations of nuclei are given in Table 1

blocked with $10 \%$ normal goat serum for $30 \mathrm{~min}$ at room temperature. Afterwards, samples were incubated overnight 
at $4^{\circ} \mathrm{C}$ with affinity-purified polyclonal anti-alarin antiserum diluted 1:350 in DAKO Antibody Diluent (DAKO, Glostrup, Denmark). For detection of the primary antibody, the slides were incubated for $40 \mathrm{~min}$ at room temperature with horseradish peroxidase-labeled polymer. Following another washing step in PBS-T, alarin-LI was visualized using DAB + solution of the DAKO ENVision System for $5 \mathrm{~min}$. Finally, the sections were dehydrated and permanently mounted using Histokitt mounting medium (Assistent, Sondheim, Germany). The specificity of the immunostaining was tested by pre-absorbtion of the affinity purified anti-alarin antiseru with $10 \mu \mathrm{M}$ of the respective peptide, for $2-3 \mathrm{~h}$ at $37^{\circ} \mathrm{C}$. Following centrifugation for $10 \mathrm{~min}$ at $13,000 \mathrm{rpm}$, serial sections were incubated with the preabsorbed serum.

\section{Image Preparation}

Pictures were taken through a Zeiss microscope equipped with AxioVision software (Zeiss, Jena, Germany). Images were adjusted when necessary for brightness and contrast using Adobe Photoshop (San Jose, CA, USA).

\section{Results}

\section{Characterization of Anti-Alarin Antiserum}

To systematically analyze the distribution of the novel neuropeptide alarin throughout the mouse brain, we performed immunohistochemistry using a polyclonal, affinity-purified anti-alarin antiserum. The antiserum was raised against alarin amino acid residues 6-24 because this sequence is not shared with GALP. Therefore, the antialarin antiserum does not show cross-reactivity for GALP and galanin (Santic et al. 2007).

Antibodies are among the most frequently used tools in basic research, but specificity and selectivity of epitope binding must be carefully tested to avoid false positive results (Lu and Bartfai 2009; Bordeaux et al. 2010). To demonstrate specificity of the anti-alarin antiserum, we first performed preabsorption experiments using C57BL/6J (wild type) brains. Preabsorption with $10 \mu \mathrm{M}$ synthetic full-length alarin peptide completely inhibited antibody binding in different brain areas (Fig. 2, d, g), whereas preabsorption with either $10 \mu \mathrm{M}$ murine GALP or galanin had no effect on antibody binding (Fig. 2a, c).

Another possibility of testing the specificity of an antibody is to ablate the cells, which express the gene of interest. To ablate alarin and GALP neurons, we bred AGALP-Cre mice to the ROSA26-DTA (R26-DTA) strain, which carries a targeted insertion of the diphtheria toxin fragment $A$ (DTA) gene in the ROSA26 locus (Brockschnieder et al. 2006) (Fig. 1b). The diphtheria toxin A chain inhibits translation by catalyzing the ADPribosylation of eukaryotic elongation factor 2 , resulting in cell death. Transcription of the R26-DTA allele terminates prematurely in R26-DTA mice, due to a loxP-flanked (floxed) strong transcriptional termination sequence. Cremediated recombination triggers DTA expression and leads to ablation of the Cre-expressing cell. Consistent with this, GALP mRNA levels were reduced by $\sim 91 \%$ in adult AGALP-Cre/R26-DTA animals (Fig. 1c). The reproductive and metabolic phenotype of AGALP-Cre/ R26-DTA mice will be described elsewhere (Mayer et al., in preparation). As shown in Fig. 2, alarin-LI is present in the hypothalamus and facial nucleus of wild-type but not AGALP-Cre/R26-DTA mice. Taken together with the preabsorption experiments, these results provide compelling evidence for the specificity of the anti-alarin antiserum.

Alarin and GALP Are Expressed in Various Brain Regions

To confirm our immunohistochemical results, we investigated the expression of alarin and GALP mRNA in alarinLI positive and negative brain areas. To do this, we performed RT-PCR analysis of total RNA extracted from different brain areas of C57BL/6J mice (Fig. 1d). The localization of the brain areas punched out is indicated in Fig. 4. Alarin mRNA was detected in the olfactory bulb, the hypothalamus, and the brainstem, but not in alain-LI negative parts of the cortex (Fig. 1d), suggesting a complex pattern of alarin expression in the murine brain. GALP peptide and mRNA are thought to be mainly expressed in the arcuate nucleus of the hypothalamus (Jureus et al. 2001; Santic et al. 2007). Consistent with this, we detected GALP mRNA in the hypothalamus (Fig. 1c, d). Unexpectedly, however, we also detected GALP mRNA in the olfactory bulb and brainstem, indicating that GALP is expressed outside of the hypothalamus.

\section{Distribution of Alarin-LI in the Mouse Brain}

The presence and intensity of alarin-LI are summarized in Figs. 3 and 4 and Table 1. A detailed description of the distribution is given below.

Telencephalon

Olfactory Bulb Alarin-LI was detected in both the main and accessory olfactory system. In the main olfactory bulb (MOB) the mitral cells as well as scattered single cells in the glomerular layer displayed alarin-LI (Figs. 3a and 4a, b). Strong diffuse labeling was observed in the accessory olfactory bulb (AOB) and the granule cell layer of the AOB (Figs. $3 \mathrm{~b}$ and $4 \mathrm{a}, \mathrm{b}$ ). In comparison to 
the main olfactory system, alarin-LI was more intense in the accessory olfactory system (Table 1).

Cortex Alarin-LI was observed in different areas of the cerebral cortex. The prelimbic cortex and the piriform cortex revealed alarin-positive neurons, and diffuse labeling was detected in the olfactory tubercle and ventral tenia tecta (Figs. $3 \mathrm{e}$ and $4 \mathrm{c}-\mathrm{j}$ ). In the cingulate cortex, pyramidal cells in layers 3 and 5 were alarin-LI positive (Figs. $3 \mathrm{f}$ and $4 \mathrm{c}-\mathrm{f}$ ). At the caudal end of the temporal lobe, alarin-LI staining was detected in the entorhinal cortex and the subiculum (Fig. 4l-n). Both areas contained alarin-LI-positive perikarya whereas diffuse labeling was observed in the ventral but not in the dorsal hippocampus.

Basal Forebrain The vertical and horizontal parts of the diagonal band of Broca were alarin-LI positive (Figs. 3c and $4 \mathrm{~d}, \mathrm{e})$. In the nucleus of the vertical limb of the diagonal band, labeling of cell bodies and diffuse staining was observed. However, in the nucleus of the horizontal limb of the diagonal band, only weak alarin-LI of perikarya was detected.

In the septal area, the lateral septal nucleus showed alarin-LI-positive cells (Figs. 3d and 4e, f). Strong staining was observed in the dorsal part of the nucleus, whereas in the lateral part, only some cell bodies were alarin-LI positive. Diffuse alarin-LI was detected in both parts of the nucleus, parallel to the medial part of the lateral ventricle.

Amygdala Along the ventral part of the hypothalamus and the optic tract, diffuse alarin-LI was detected. It showed high intensity in the area of the medial amygdala, becoming weak again in the region of the cortical amygdaloid nucleus. In the medial amygdala, alarin-LI-positive cell bodies as well as diffuse labeling within the whole nucleus occurred (Figs. 31 and $4 \mathrm{~h}-\mathrm{j}$ ). In the cortical amygdaloid nucleus, weak labeling of cells bodies was observed (Figs. 4h-j).

\section{Diencephalon}

Alarin-LI was found throughout the diencephalon, but labeling was strongest in the hypothalamic area.

Epithalamus In the medial habenular nucleus, strong but diffuse alarin-LI was observed in and around cell bodies (Figs. $3 \mathrm{~g}$ and $4 \mathrm{~h}-\mathrm{j}$ ). In contrast, no alarin-LI-positive cells were detected in the lateral habenular nucleus.

Thalamus In the thalamic area, only the paraventricular thalamic nucleus revealed alarin-LI (Figs. $3 \mathrm{~g}$ and $4 \mathrm{f}-\mathrm{i}$ ).
Alarin-LI-positive cell bodies were detected adjacent to the third ventricle. However, compared to the intensity of labeling in the neighbouring medial habenular nucleus, staining in the paraventricular thalamic nucleus appeared weak (Figs. 3g and 4h, Table 1).

Hypothalamus Intense but diffuse alarin-LI was predominantly detected in the ventral part of the hypothalamus and adjacent to the third ventricle. In the preoptic area, alarin-LI was found in the median preoptic nucleus, the ventromedial preoptic nucleus, the anteroventral periventricular nucleus, the medial preoptic nucleus, and the magnocellular preoptic nucleus (Figs. 3h and 4e, f). All nuclei were positive for cell bodies, and the intensity of the labeling, except in the magnocellular nucleus, was strong.

Caudal to the preoptic area, intense alarin-LI was observed in the supraoptic nucleus, the suprachiasmatic nucleus, the ventromedial hypothalamic nucleus, the arcuate nucleus, the dorsal tuberomammilary nucleus, and the dorsomedial hypothalamic nucleus (Figs. $3 \mathrm{i}, \mathrm{j}$ and $4 \mathrm{~g}-\mathrm{k}$ ). Furthermore, diffuse labeling and some alarin-LI-positive cells were seen in the periventricular hypothalamic nucleus and the lateral hypothalamic area. In the paraventricular hypothalamic nucleus, some magnocellular secretory cells were positive for alarin-LI as were some small single cells in the optic tract (Fig. 3k).

\section{Mesencephalon}

Diffuse alarin-LI was observed in the interpeduncular nucleus, showing high labeling intensity (Figs. $3 \mathrm{~m}$ and $41, \mathrm{~m})$. Caudally, the pontine nuclei revealed strong alarinLI in medium-sized cells and diffuse staining around the positive cells (Figs. 30 and $4 \mathrm{~m}$ ). Lateral to the pontine nuclei, small single cells were detected in the middle cerebellar peduncle, and labelling of medium intensity was observed in the lateral lemniscus (Figs. $3 n$ and $4 n$ ). In the periaqueductal gray, weak alarin-LI of cell bodies was observed along the aqueduct (Fig. 4l-n).

Pons

Beginning in the mesencephalon, the trigeminal tract showed alarin-LI of different intensities (Table 1). Whereas the sensory and motor root of the trigeminal nerve revealed only some small alarin-positive cells, large multipolar neurons were detected in the parvicellular motor trigeminal nucleus, the principal sensory trigeminal nucleus, and the motor trigeminal nucleus (Figs. $3 p$ and $4 n, o$ ). Adjacent to the fourth ventricle, the locus coeruleus, the dorsal tegmental nucleus, and the parabranchial nucleus were all alarin-LI positive (Figs. 3q and 4o). Whereas the dorsal 
tegmental nucleus and the parabranchial nucleus revealed only weak labeling of cell bodies, the locus coeruleus showed a higher intensity of alarin-LI.

\section{Medulla Oblongata}

In the dorsal medulla, alarin-LI was detected in the medial vestibular nucleus, adjacent to the fourth ventricle (Fig. 4p). On the ventral aspect, the oliviary complex and the nucleus of the trapezoid body revealed alarin-LI-positive cells as well as diffuse alarin-LI labeling (Figs. $3 \mathrm{r}$ and 40 ). In the trapezoid body and superior olive, only cell bodies were alarin-LI positive, whereas in the perioliviary region, some cell bodies and diffuse staining around the perikarya were observed. In the lateral medulla, small cell bodies were detected in the cochlear nucleus and spinal trigeminal tract (Figs. 3s and 4o, p). Caudally, the facial nucleus showed clear, alarin-LI-positive neurons of high labelling intensity (Figs. 3t and 4p).

\section{Circumventricular Organs and Ventricle}

The epithelial layer of the choroid plexus of the lateral ventricles revealed alarin-LI positve cells (Fig. 3g). Only one of the circumventricular organs of the mouse showed intense alarinergic innervation. In the vascular organ of the lamina terminalis, strong diffuse alarin labelling was detected (Figs. 3h and 4e). The other circumventricular organs, except the median eminence, were alarin negative. In the median eminence, weak diffuse alarinergic innervation, but no alarin-positive cell bodies, was detected.

\section{Discussion}

Here, we demonstrate for the first time that alarin-LI is present in a variety of nuclei throughout the mouse brain. Alarin is detected in similar brain regions as other members of the galanin peptide family. For instance, in the telencephalon, alarin-LI and galanin are both found in the accessory olfactory bulb, the diagonal band of Broca, the lateral septal nucleus, and in the medial amygdala (Perez et al. 2001). Especially in the hypothalamic area, the expression patterns of both peptides are quite similar (Perez et al. 2001), raising the possibility that some neurons in these regions might express more than one peptide of the galanin peptide family. Immunoreactivity for both peptides was detected in the medial preoptic nucleus, the periventricular nucleus, the paraventricular nucleus, the supraoptic nucleus, the dorsomedial nucleus, the premammilary nucleus, the lateral hypothalamic area, the ventromedial nucleus, and the arcuate nucleus (Perez et al. 2001). However, in the thalamus, for example, alarin-LI was present only in the paraventricular thalamic nucleus, whereas galanin immunoreactivity was detected in a variety of thalamic brain areas (Perez et al. 2001).

In the murine brain, the distribution of GALP mRNA expression was detected in the arcuate nucleus of the hypothalamus (Jureus et al. 2001). In situ hybridization, however, did not differentiate between the GALP and alarin splice variant. The alarin-LI in cell bodies of the arcuate nucleus detected in the present study is consistent with the mRNA expression of the GALP gene previously observed in those same cells (Jureus et al. 2001). In the rat brain, GALP mRNA expression and GALP-LI were also detected in the arcuate nucleus, indicating the expression of both splice variants in this region.

Localization of alarin-LI to the locus coeruleus of mice has also been reported in the rat brain (Van Der Kolk et al. 2010), indicating that there might be a similar distribution of alarin in both species. Since the distribution of GALP by immunohistochemistry has only been investigated in the rat brain so far (Takatsu et al. 2001), we can only compare the alarin-LI in mice to the localization of GALP-LI in rats.

An overlap in the expression of the splice variants GALP and alarin seems to be present in the medial preoptic area, the lateral septal nucleus, the paraventricular hypothalamic nucleus, the periventricular hypothalamic nucleus, and the arcuate nucleus (Takatsu et al. 2001). Because there are several regions that do not overlap between GALP and alarin, the two peptides may also have distinct functions and receptors.

So far, expression of GALP/alarin mRNA, detected by in situ hybridization, has only been reported in the hypothalamus but not in other regions of the murine brain (Jureus et al. 2001). We were also not able to detect GALP and alarin mRNA in whole brain preparations by classical RT-PCR. Although we have punched out alarin-LI positive regions brain, we needed a nested RT-PCR to detect GALP and alarin mRNA in these regions, indicating that the mRNA expression levels of GALP and alarin are very low. Because of the low expression levels of these two peptides, it will be extremely difficult to distinguish their expression by in situ hybridization with exon specific probes.

The diffuse staining of the extracellular matrix is a phenomenon, which can only be observed in regions containing alarin-LI-positive cell bodies (Figs. 2 and 3). In brain regions where no alarin-LI-positive cells are detected, no diffuse background staining was observed. Therefore, we hypothesize, that alarin is secreted from the cells into the extracellular matrix, leading to a diffuse staining pattern of the antibody. However, we also cannot exclude that there is some diffuse unspecific background staining present.

Recently, i.c.v. injection of alarin into the lateral ventricle of rats was shown to activate the expression of 



G

4 Fig. 3 a, b. Olfactory system. Alarin-LI-positive cells were detected in the mitral cell layer, the glomerular cell layer, and the accessory olfactory bulb. c, d. Basal forebrain. The nuclei of the vertical and horizontal limb of the diagonal band and the lateral septal nucleus revealed alarin-LI. e, f. In the cortex alarin-LI-positive cells were detected in the piriform and cingulate cortex. $\mathbf{g}$ In the thalamic area and third ventricle, the choroid plexus and medial habenular nucleus show strong alarin-LI and weak alarin-LI-positive cells in the paraventricular thalamic nucleus. $\mathbf{h}-\mathbf{j}$ Prepotic area. Intense alarinLI-positive cells and diffuse labeling are detected in the medial preoptic area, the median preoptic nucleus, the vascular organ of the lamina terminalis, the suprachiasmatic nucleus, the supraoptic nucleus, and in the lateral hypothalamic area. Only single cells are detected in the optic tract. $\mathbf{k}$ Alarin-LI in magnocellular secretory cells of the paraventricular hypothalamic nucleus. I Intense alarin-LI of cells detected in the medial amygdala and small single cells in the optic tract. m Diffuse alarin-LI in the interpeduncular nucleus. n Small single cells positive for alarin-LI indicated in the motor root of the trigeminal nerve and middle cerebellar peduncle. In the lateral lemniscus, cell bodies revealed to be positive for alarin-LI. o Strong alarin-LI of medium-sized cells detected in pontine nuclei. p AlarinLI-positive neurons in the motor trigeminal nucleus, the motor root of the trigeminal nerve, and the parvicellular motor trigeminal nucleus. $\mathbf{q}$ In the pond, the locus coeruleus showed alarin-LI-positive neurons. $\mathbf{r}$ Alarin-LI-positive neurons in the nucleus of the trapezoid body. s In the chochlear nucleus, small single cells were alarin-LI positive. $t$ Alarin-LI-positive neurons in the facial nucleus. Abbreviations of nuclei are given in Table 1. Abbreviations not included in Table 1: $3 \mathrm{~V}$ third ventricle, $D 3 V$ third ventricle dorsal part, lfp longitudinal fasciculus of the pons, scale bars $=100 \mu \mathrm{m}$

the immediate-early gene fos in rat brain (Van Der Kolk et al. 2010). Because fos activation is a powerful tool to identify neuronal activation (Kovacs 2008), it is most likely that alarin-recognizing receptors are expressed in regions where stimulation of fos expression occurs after alarin treatment. In comparison to the localization of alarin-LI in this study, fos immunoreactivty is detected in similar brain regions. Alarin-LI and alarin-induced fos expression are observed in the telencephalon in the medial preoptic nucleus, the diagonal band of Broca, the paraventricular hypothalamic nucleus, the lateral hypothalamus, the dorsomedial nucleus, the lateral septal nucleus, and the paraventricular nucleus of the thalamus. In extra-diencephalic structures, an overlap of alarin-LI and alarin-induced fos expression seems to be present in the central gray, the locus coeruleus, and in olive nuclei, whereas in raphe nuclei, the nucleus prepositus, the dorsal vestibular nucleus, and the dorsal motor nucleus of the vagus, only fos-immunoreactive cells are detected. These data indicate that alarin activates neurons in or adjacent to brain nuclei containing alarin-LIpositive cells. The activation of $f o s$ also indicates the presence of alarin receptors in these cells.

\section{Olfaction}

One key finding of this study is the detection of alarin-LI in several brain areas dedicated to the sense of smell. The olfactory system in rodents is important for reproduction, consistent with a putative function of alarin in modulating reproductive physiology. The rodent nose houses two distinct sensory epithelia that are thought to be specialized in detecting different classes of chemosensory cues (reviewed by (Tirindelli et al. 2009). Alarin-LI was detected at different levels in both the main and accessory olfactory system.

\section{Alarin-LI in Brain Areas that Relay Odor Information}

We detected alarin-LI in the MOB, which receives centrifugal input from many different brain areas (Shipley et al. 2004). Specifically, alarin-LI was observed in the mitral cells, which are the first-order relay neurons receiving direct input from olfactory sensory neurons in the main olfactory epithelium (MOE). These data suggest a potential neuromodulatory function of alarin in response to odor signals from the MOE. In addition, alarin-LI was detected in the granule cell layer of
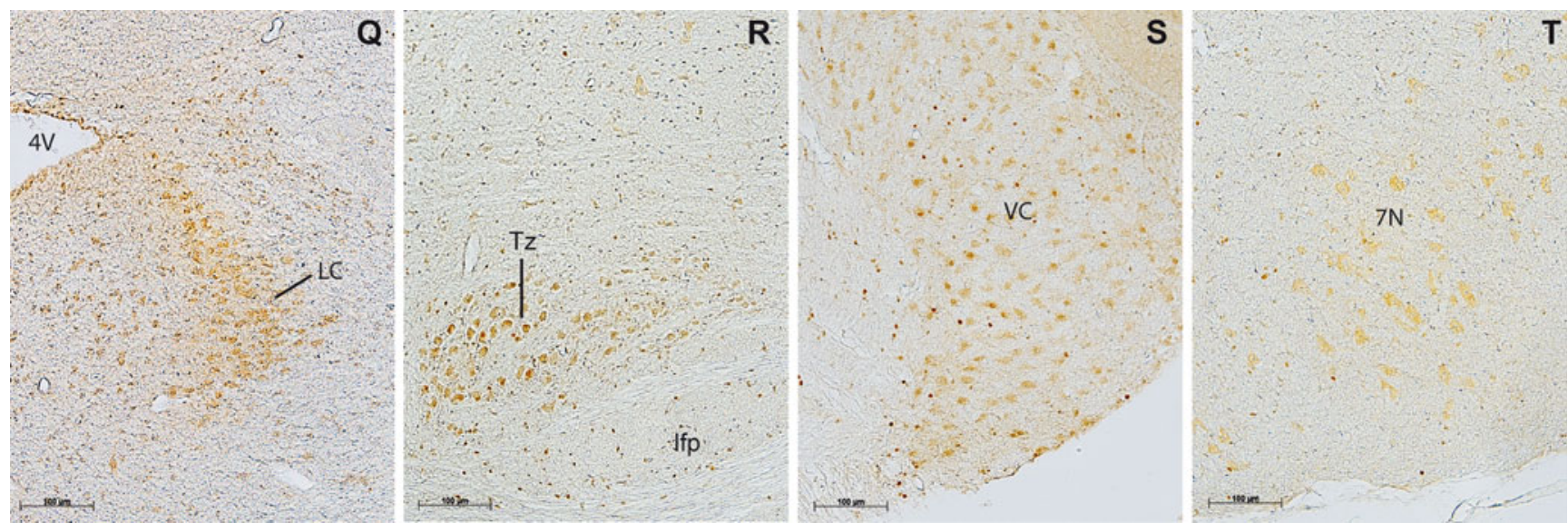

Fig. 3 (continued) 



Fig. 4 a-p Composite schematic drawings of coronal sections showing the distribution of alarin-LI in murine brain. Dots indicate alarin-LI-positive brain regions. Small dots indicate brain nuclei where

only small single cells are positive for alarin-LI. Abbreviations used in the figure are included in Table 1. Squares indicate the areas punched out for RT-PCR studies

the MOB. Information from the MOB is transmitted to the primary olfactory cortex (piriform cortex) and to the olfactory amygdala (ACO), which impinges onto neuroendocrine target neurons in the hypothalamus such as $\mathrm{GnRH}$ neurons (Boehm et al. 2005; Yoon et al. 2005). Strikingly, alarin-LI was detected both in the piriform cortex and the ACO, raising the possibility that alarin might be released in response to certain odor cues. 

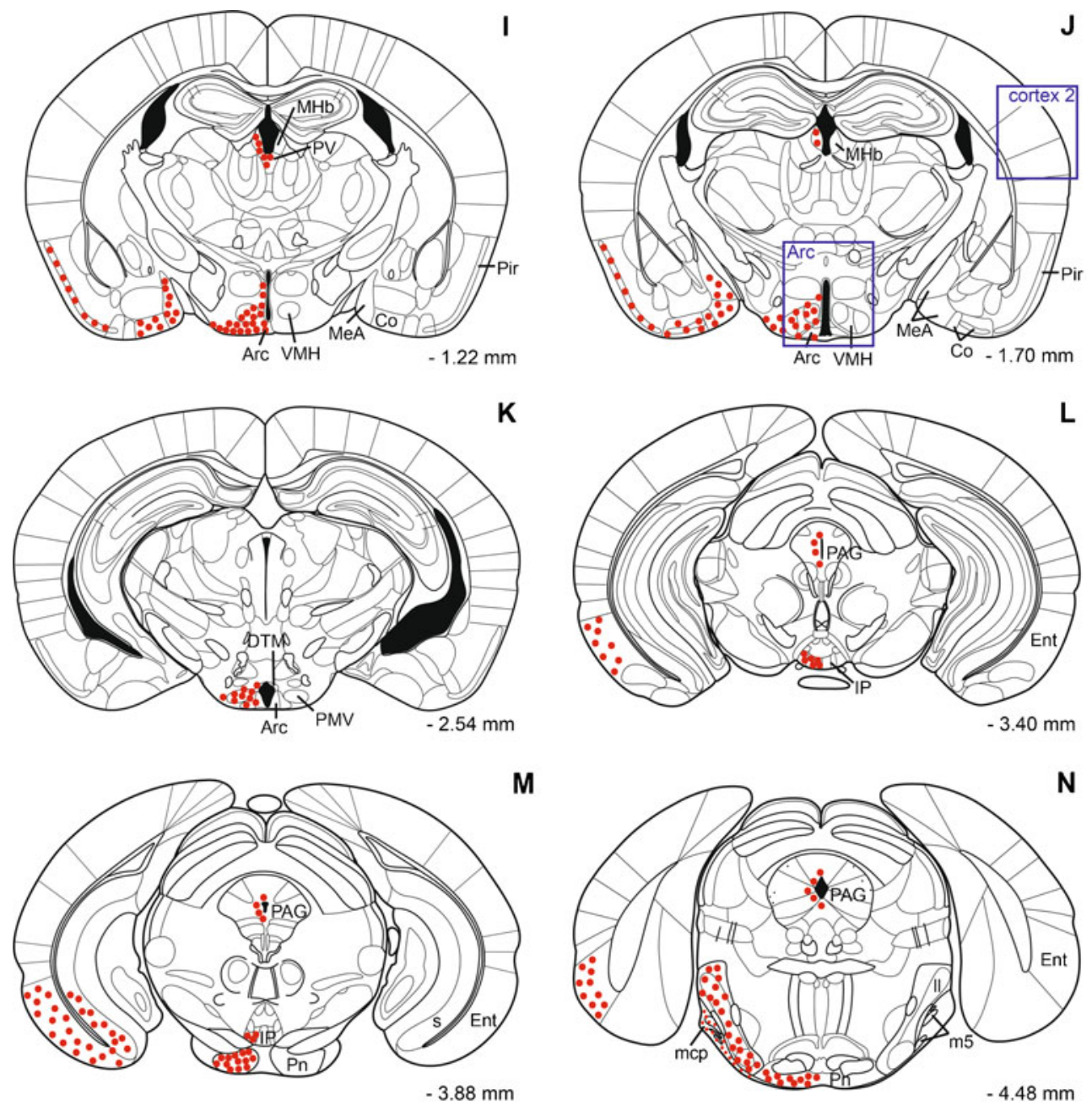

M
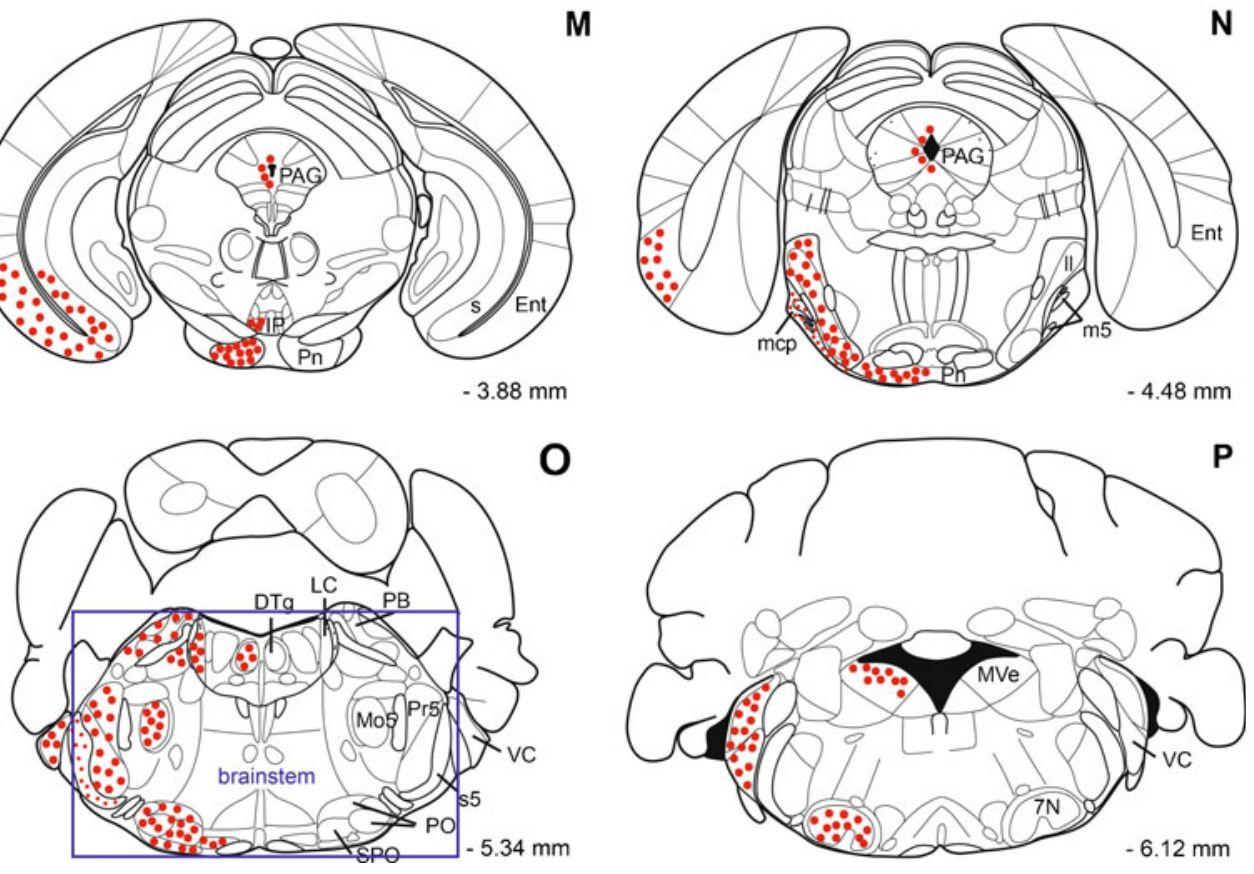

Fig. 4 (continued)

Alarin-LI in Pheromone Relay Areas

Intense alarin-LI was also found in the AOB, which receives direct input from sensory neurons in the vomeronasal organ (VNO). The VNO is spatially segregated from the MOE and is specialized in the detection of pheromones (Halpern and Martinez-Marcos 2003). Information from the AOB is transmitted to the vomeronasal amygdala, which is specialized in relaying socially relevant signals from the VNO to the hypothalamus (Halpern and Martinez-Marcos
2003). We found both alarin-LI-positive fibers and perikarya in the medial amygdala, raising the possibility that alarin might be released in response to certain pheromones. In addition, alarin-LI is expressed in the central amygdaloid nucleus, functioning as an output area of the amygdala and connecting it to brainstem nuclei (including the locus coeruleus) that mediate peripheral effects of fear responses. Taken together, the distribution of alarin-LI indicates that the peptide may be secreted in response to chemosensory cues relevant for reproduction and metabolism. 
Table 1 Abbreviations and summary of alarin-LI in murine brain nuclei

\begin{tabular}{ll}
\hline Region & Abbreviation
\end{tabular}

Accessory olfactory bulb

Anteroventral periventicular nucleus

Arcuate nucleus of the hypothalamus

Cingulate cortex

Cochlear nucleus

Cortical amygdaloid nucleus

Dorsal tuberomammilary nucleus

Dorsal tegmental nucleus

Dorsomedial hypothalamic nucleus

Entorhinal cortex

Facial nucleus

Glomerular layer of the olfactory bulb

Granule cell layer of the accessory olfactory bulb

Interpeduncular nucleus

Lateral hypothalamic area

Lateral lemniscus

Lateral septal nucleus

Locus coeruleus

Magnocellular preoptic nucleus

Medial amygdala

Medial habenular nucleus

Medial preoptic area

Medial preoptic nucleus

Medial vestibular nucleus

Median preoptic nucleus

Middle cerebellar peduncle

Mitral cell layer of the olfactory bulb

Motor root of the trigeminal nerve

Motor trigeminal nucleus

Nucleus of the horizontal limb of the diagonal band

Nucleus of the trapezoid body

Nucleus of the vertical limb of the diagonal band

Olfactory tubercle

Optic tract

Parabranchial nucleus

Paraventricular hypothalamic nucleus

Paraventricular thalamic nucleus

Parvicellular motor trigeminal nucleus

Periaqueductal gray

Perioliviary region

Periventricular hypothalamic nucleus

Piriform cortex

Pontine nuclei

Prelimbic cortex

Premammillary nucleus, ventral part

Principal sensory trigeminal nucleus

Sensory root of the trigeminal nerve

Spinal trigeminal tract

Subiculum

\begin{tabular}{|c|c|}
\hline AOB & +++ \\
\hline AVPe & +++ \\
\hline Arc & +++ \\
\hline $\mathrm{Cg}$ & + \\
\hline $\mathrm{VC}$ & ++ \\
\hline $\mathrm{Co}$ & + \\
\hline DTM & ++ \\
\hline DTg & + \\
\hline $\mathrm{DMH}$ & +++ \\
\hline Ent & + \\
\hline $7 \mathrm{~N}$ & ++ \\
\hline Gl & ++ \\
\hline GlA & +++ \\
\hline IP & ++ \\
\hline LA & + \\
\hline 11 & ++ \\
\hline LS & ++ \\
\hline $\mathrm{LC}$ & ++ \\
\hline MCPO & + \\
\hline $\mathrm{MeA}$ & ++ \\
\hline $\mathrm{MHb}$ & ++ \\
\hline MPA & +++ \\
\hline MPO & ++ \\
\hline MVe & ++ \\
\hline $\mathrm{MnPO}$ & +++ \\
\hline mcp & + \\
\hline $\mathrm{Mi}$ & ++ \\
\hline $\mathrm{m} 5$ & + \\
\hline Mo5 & ++ \\
\hline HDB & + \\
\hline $\mathrm{Tz}$ & ++ \\
\hline VDB & ++ \\
\hline $\mathrm{Tu}$ & + \\
\hline opt & + \\
\hline PB & + \\
\hline $\mathrm{Pa}$ & + \\
\hline PV & + \\
\hline PC5 & + \\
\hline PAG & +- \\
\hline $\mathrm{PO}$ & + \\
\hline $\mathrm{Pe}$ & + \\
\hline Pir & + \\
\hline Pn & +++ \\
\hline PrL & ++ \\
\hline PMV & +++ \\
\hline $\operatorname{Pr} 5$ & ++ \\
\hline s5 & + \\
\hline Sp5 & + \\
\hline S & + \\
\hline
\end{tabular}


Table 1 (continued)

\begin{tabular}{lll}
\hline Region & Abbreviation & Intensity $^{\text {a }}$ \\
\hline Superior olive & SPO & ++ \\
Suprachiasmatic nucleus & SCh & +++ \\
Supraoptic nucleus & SO & +++ \\
Vascular organ of the lamina terminalis & VOLT & VH \\
Ventral hippocampus & VMH & +++ \\
Ventromedial hypothalamic nucleus & VMPO \\
Ventromedial preoptic nucleus & & +++ \\
\hline
\end{tabular}

${ }^{a}$ Brain regions showed strong $(+++)$, medium $(++)$ or weak $(+)$ intensity of alarin-LI

\section{Reproduction}

Alarin impinges on the hypothalamic-pituitary-gonadal (hpg) axis and influences LH secretion (Van Der Kolk et al. 2010; Boughton et al. 2010). Our novel data may illuminate a neural substrate potentially underlying these effects. LH secretion is controlled by a small subset of hypothalamic neurons that produce and secrete GnRH. $\mathrm{GnRH}$ neurons mediate central control of reproductive physiology in vertebrates and communicate with a large number of neurons in other parts of the brain to integrate a variety of signals important for reproduction (Boehm et al. 2005; Yoon et al. 2005; Herbison 2006). GnRH neurons receive information from at least 10,000 neurons in 12 different brain areas (Boehm et al. 2005). Interestingly, some of these brain areas (e.g., in the hypothalamus and thalamus) with neurons upstream of GnRH neurons display alarin-LI, suggesting that alarin effects on GnRH neurons might be direct. Consistent with this, alarin was shown to stimulate GnRH release in both hypothalamic explants and a hypothalamic cell line (Boughton et al. 2010). Because alarin lacks homology to galanin and does not bind to galanin receptors (Santic et al. 2007; Boughton et al. 2010), alarin effects on LH secretion are probably mediated via an unknown receptor. Future studies will need to identify the alarin receptor and determine whether it is expressed in GnRH neurons.

\section{Metabolism}

Alarin injection into the brain stimulates feeding behavior and increases body weight (Van Der Kolk et al. 2010; Boughton et al. 2010). The feeding center in the hypothalamus resides in the arcuate nucleus (Cone 2005; Horvath 2005). Many different neuropeptides that influence feeding behavior and body weight are expressed by arcuate nucleus neurons, including proopiomelanocortin (POMC), $\alpha$ melanocyte-stimulating hormone $(\alpha-\mathrm{MSH})$, neuropeptide Y (NPY), and agouti-related protein (AgRP) (Cone 2005; Horvath 2005). A well-established model neural circuit underlying feeding behavior comprises NPY/AgRP and POMC/ $\alpha$-MSH neurons: Activation of POMC neurons by leptin triggers the release of $\alpha-\mathrm{MSH}$ from POMC axon terminals and leads to suppressed food intake and increased energy expenditure. Simultaneously, leptin suppresses the activity of arcuate nucleus NPY/ AgRP neurons, which otherwise would antagonize the $\alpha$-MSH effect through the release of AgRP (Horvath 2005). Consistent with alarin's role in influencing metabolism, alarin-LI was detected in the arcuate nucleus. This is also where GALP is expressed (see above). Only a subset of arcuate nucleus neurons displays alarin-LI. It is not known whether the same or different sets of neurons co-express alarin and GALP. It is also not known whether alarin is expressed in $\mathrm{POMC} / \alpha$ MSH or NPY/AgRP neurons.

In conclusion, the present study clearly establishes alarin as a multifunctional neuropeptide. As is the rule for neuropeptides, alarin is expressed in discrete areas of the brain. Because these loci are known to regulate metabolism, reproduction, and behaviour, alarin is poised to be added to the list of multifunctional regulatory peptides.

Acknowledgments We are indebted to Olaf Pongs for continuous support. This work was supported by a research grant of the Paracelsus Medical University (grant number 05/01/003), the "Verein zur Förderung Pädiatrischer Forschung und Fortbildung, Salzburg", a research grant of the Austrian Research Promotion Agency (FFG, 822782/THERAPEP), and a research grant by the Deutsche Forschungsgemeinschaft (DFG): BO1743/2 (to U. Boehm). We thank Irm Hermans-Borgmeyer (ZMNH transgenic service group) for pronucleus injection. 
Open Access This article is distributed under the terms of the Creative Commons Attribution Noncommercial License which permits any noncommercial use, distribution, and reproduction in any medium, provided the original author(s) and source are credited.

\section{References}

Boehm U, Zou Z, Buck LB (2005) Feedback loops link odor and pheromone signaling with reproduction. Cell 123:683-695

Bordeaux J, Welsh A, Agarwal S, Killiam E, Baquero M, Hanna J, Anagnostou V, Rimm D (2010) Antibody validation. Biotechniques 48:197-209

Boughton CK, Patterson M, Bewick GA, Tadross JA, Gardiner JV, Beale KE, Chaudery F, Hunter G, Busbridge M, Leavy EM, Ghatei MA, Bloom SR, Murphy KG (2010) Alarin stimulates food intake and gonadotrophin release in male rats. Br J Pharmacol 161:601-613

Brockschnieder D, Pechmann Y, Sonnenberg-Riethmacher E, Riethmacher D (2006) An improved mouse line for Creinduced cell ablation due to diphtheria toxin A, expressed from the Rosa26 locus. Genesis 44:322-327

Cone RD (2005) Anatomy and regulation of the central melanocortin system. Nat Neurosci 8:571-578

Enigk RE, Maimone MM (1999) Differential expression and developmental regulation of a novel alpha-dystrobrevin isoform in muscle. Gene 238:479-488

Grabowski PJ, Black DL (2001) Alternative RNA splicing in the nervous system. Prog Neurobiol 65:289-308

Halpern M, Martinez-Marcos A (2003) Structure and function of the vomeronasal system: an update. Prog Neurobiol 70:245-318

Herbison AE (2006) Physiology of the gonadotropin-releasing hormone neuronal network. Elsevier Academic Press, St. Louis

Horvath TL (2005) The hardship of obesity: a soft-wired hypothalamus. Nat Neurosci 8:561-565

Jureus A, Cunningham MJ, Li D, Johnson LL, Krasnow SM, Teklemichael DN, Clifton DK, Steiner RA (2001) Distribution and regulation of galanin-like peptide (GALP) in the hypothalamus of the mouse. Endocrinology 142:5140-5144

Kovacs KJ (2008) Measurement of immediate-early gene activationc-fos and beyond. J Neuroendocrinol 20:665-672
Lang R, Gundlach AL, Kofler B (2007) The galanin peptide family: receptor pharmacology, pleiotropic biological actions, and implications in health and disease. Pharmacol Ther 115:177-207

Lu X, Bartfai T (2009) Analyzing the validity of GalR1 and GalR2 antibodies using knockout mice. Naunyn Schmiedebergs Arch Pharmacol 379:417-442

Ohtaki T, Kumano S, Ishibashi Y, Ogi K, Matsui H, Harada M, Kitada C, Kurokawa T, Onda H, Fujino M (1999) Isolation and cDNA cloning of a novel galanin-like peptide (GALP) from porcine hypothalamus. J Biol Chem 274:37041-37045

Perez SE, Wynick D, Steiner RA, Mufson EJ (2001) Distribution of galaninergic immunoreactivity in the brain of the mouse. J Comp Neurol 434:158-185

Santic R, Fenninger K, Graf K, Schneider R, Hauser-Kronberger C, Schilling FH, Kogner P, Ratschek M, Jones N, Sperl W, Kofler B (2006) Gangliocytes in neuroblastic tumors express alarin, a novel peptide derived by differential splicing of the galanin-like peptide gene. J Mol Neurosci 29:145-152

Santic R, Schmidhuber SM, Lang R, Rauch I, Voglas E, Eberhard N, Bauer JW, Brain SD, Kofler B (2007) Alarin is a vasoactive peptide. Proc Natl Acad Sci U S A 104:10217-10222

Shipley MT, Ennis M, Puche A (2004) Olfactory system. Elsevier Academic Press, San Diego

Takatsu Y, Matsumoto H, Ohtaki T, Kumano S, Kitada C, Onda H, Nishimura O, Fujino M (2001) Distribution of galanin-like peptide in the rat brain. Endocrinology 142:1626-1634

Tirindelli R, Dibattista M, Pifferi S, Menini A (2009) From pheromones to behavior. Physiol Rev 89:921-956

Van Der Kolk N, Madison FN, Mohr M, Eberhard N, Kofler B, Fraley GS (2010) Alarin stimulates food intake in male rats and LH secretion in castrated male rats. Neuropeptides 44:333-340

Warming S, Costantino N, Court DL, Jenkins NA, Copeland NG (2005) Simple and highly efficient BAC recombineering using galK selection. Nucleic Acids Res 33:e36

Wen S, Schwarz JR, Niculescu D, Dinu C, Bauer CK, Hirdes W, Boehm U (2008) Functional characterization of genetically labeled gonadotropes. Endocrinology 149:2701-2711

Yoon H, Enquist LW, Dulac C (2005) Olfactory inputs to hypothalamic neurons controlling reproduction and fertility. Cell 123:669-682 